$$
\begin{aligned}
& \text { N.64 } \\
& 83210
\end{aligned}
$$

NASA/CR-201750

ICASE Report No. 97-55

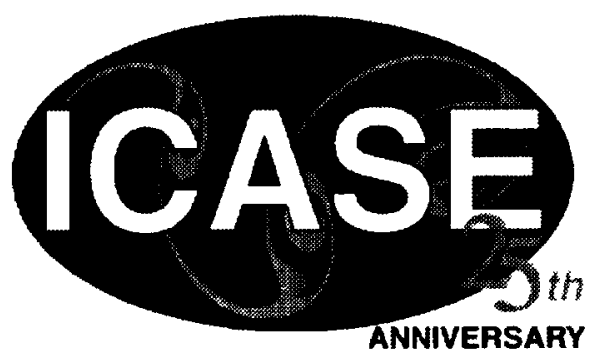

\title{
Simulation of a Controlled Airfoil with Jets
}

\author{
Brian G. Allan, Maurice Holt, and Andrew Packard
}




\section{The NASA STI Program Office ... in Profile}

Since its founding, NASA has been dedicated to the advancement of aeronautics and space science. The NASA Scientific and Technical Information (STI) Program Office plays a key part in helping NASA maintain this important role.

The NASA STI Program Office is operated by Langley Research Center, the lead center for NASA's scientific and technical information. The NASA STI Program Office provides access to the NASA STI Database, the largest collection of aeronautical and space science STI in the world. The Program Office is also NASA's institutional mechanism for disseminating the results of its research and development activities. These results are published by NASA in the NASA STI Report Series, which includes the following report types:

- TECHNICAL PUBLICATION. Reports of completed research or a major significant phase of research that present the results of NASA programs and include extensive data or theoretical analysis. Includes compilations of significant scientific and technical data and information deemed to be of continuing reference value. NASA counter-part or peer-reviewed formal professional papers, but having less stringent limitations on manuscript length and extent of graphic presentations.

- TECHNICAL MEMORANDUM. Scientific and technical findings that are preliminary or of specialized interest, e.g., quick release reports, working papers, and bibliographies that contain minimal annotation. Does not contain extensive analysis.

- CONTRACTOR REPORT. Scientific and technical findings by NASA-sponsored contractors and grantees.
- CONFERENCE PUBLICATIONS. Collected papers from scientific and technical conferences, symposia, seminars, or other meetings sponsored or co-sponsored by NASA.

- SPECIAL PUBLICATION. Scientific, technical, or historical information from NASA programs, projects, and missions, often concerned with subjects having substantial public interest.

- TECHNICAL TRANSLATION. Englishlanguage translations of foreign scientific and technical material pertinent to NASA's mission.

Specialized services that help round out the STI Program Office's diverse offerings include creating custom thesauri, building customized databases, organizing and publishing research results ... even providing videos.

For more information about the NASA STI Program Office, you can:

- Access the NASA STI Program Home Page at http://www.sti.nasa.gov/STIhomepage.html

- Email your question via the Internet to help@sti.nasa.gov

- Fax your question to the NASA Access Help Desk at (301) 621-0134

- Phone the NASA Access Help Desk at (301) 621-0390

- Write to:

NASA Access Help Desk

NASA Center for AeroSpace Information 800 Elkridge Landing Road

Linthicum Heights, MD 21090-2934 
NASA/CR-201750

ICASE Report No. 97-55

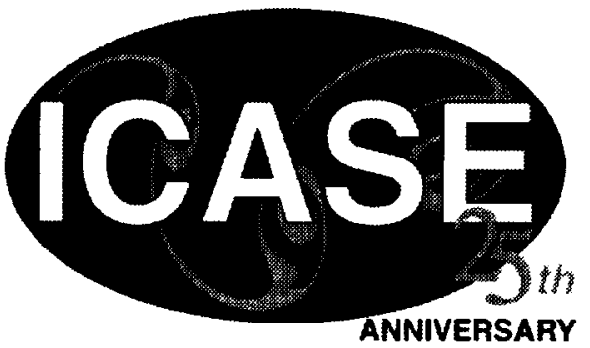

\section{Simulation of a Controlled Airfoil with Jets}

Brian G. Allan

ICASE

and

Maurice Holt and Andrew Packard

University of California, Berkeley

Institute for Computer Applications in Science and Engineering

NASA Langley Research Center

Hampton, VA

Operated by Universities Space Research Association

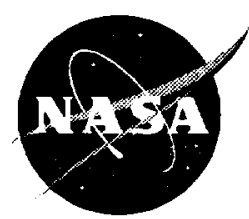

National Aeronautics and

Space Administration

Langley Research Center

Hampton, Virginia 23681-2199

October 1997 
Available from the following:

NASA Center for AeroSpace Information (CASI)

800 Elkridge Landing Road

Linthicum Heights, MD 21090-2934

(301) 621-0390
National Technical Information Service (NTIS) 5285 Port Royal Road

Springfield, VA 22161-2171

(703) $487-4650$ 


\title{
SIMULATION OF A CONTROLLED AIRFOIL WITH JETS
}

\author{
BRIAN G. ALLAN *, MAURICE HOLT ${ }^{\dagger}$, AND ANDREW PACKARD $\ddagger$
}

\begin{abstract}
Numerical simulations of a two-dimensional airfoil, controlled by an applied moment in pitch and an airfoil controlled by jets, were investigated. These simulations couple the Reynolds-averaged NavierStokes equations and Euler's equations of rigid body motion, with an active control system. Controllers for both systems were designed to track altitude commands and were evaluated by simulating a closedloop altitude step response using the coupled system. The airfoil controlled by a pitching moment used an optimal state feedback controller. A closed-loop simulation, of the airfoil with an applied moment, showed that the trajectories compared very well with quasi-steady aerodynamic theory, providing a measure of validation. The airfoil with jets used a controller designed by robust control methods. A linear plant model for this system was identified using open-loop data generated by the nonlinear coupled system. A closedloop simulation of the airfoil with jets, showed good tracking of an altitude command. This simulation also showed oscillations in the control input as a result of dynamics not accounted for in the control design. This research work demonstrates how computational fluid dynamics, coupled with rigid body dynamics, and a control law can be used to prototype control systems in problematic nonlinear flight regimes.
\end{abstract}

Key words. Navier-Stokes equations, numerical simulation, feedback control, nonlinear flight regime, jets, coupled fluid dynamics, rigid body dynamics, and controls

Subject classification. Applied Numerical Mathematics

1. Introduction. In nonlinear flight regimes, the interaction between fluids, body dynamics, and controls can critically effect the performance of an aircraft. By coupling these disciplines one can computationally investigate the problematic nonlinear portions of the flight envelope. However, simulation models which rely on empiricism or linearity assumptions may give misleading results when applied to nonlinear flight regimes. To capture the nonlinear nature of the flow field, higher-ordered models need to be considered. In this investigation, the Reynolds-averaged Navier-Stokes (RANS) equations are coupled with Euler's equations of motion, along with an active control system.

The application which will provide the impetus for this work is the controlled landing of a highperformance powered-lift aircraft. This flight regime is currently problematic due to the difficulties in obtaining an adequate representation of the stability derivatives. In turn, use of a poor plant description to design the control system can lead to the loss of aircraft and pilot. The results of this research would begin to provide a control system designer with a means of computationally testing the control system in these problematic nonlinear flight regimes, thus helping to avoid these costly errors.

Previous efforts have validated the component problems of unsteady fluid dynamics [1], the specified

\footnotetext{
*Institute for Computer Applications in Science and Engineering, Mail Stop 403, NASA Langley Research Center, Hampton, VA 23681-0001 (Email: allan@icase.edu). This research was supported by the National Aeronautics and Space Administration under NASA Contract No. NAS1-19480 while the first author was in residence at the Institute for Computer Applications in Science and Engineering (ICASE), NASA Langley Research Center, Hampton, VA 23681-0001 and by the NASA Graduate Student Researchers Program in High Performance Computing and Communication, Fellowship number NGT-51030 and by NASA Ames Consortium number NCA2-782 and NCC2-5035.

†Department of Mechanical Engineering, University of California, Berkeley, Berkeley, CA 94720

¥Department of Mechanical Engineering, University of California, Berkeley, Berkeley, CA 94720 (Email: pack@erg.me.berkeley.edu).
} 


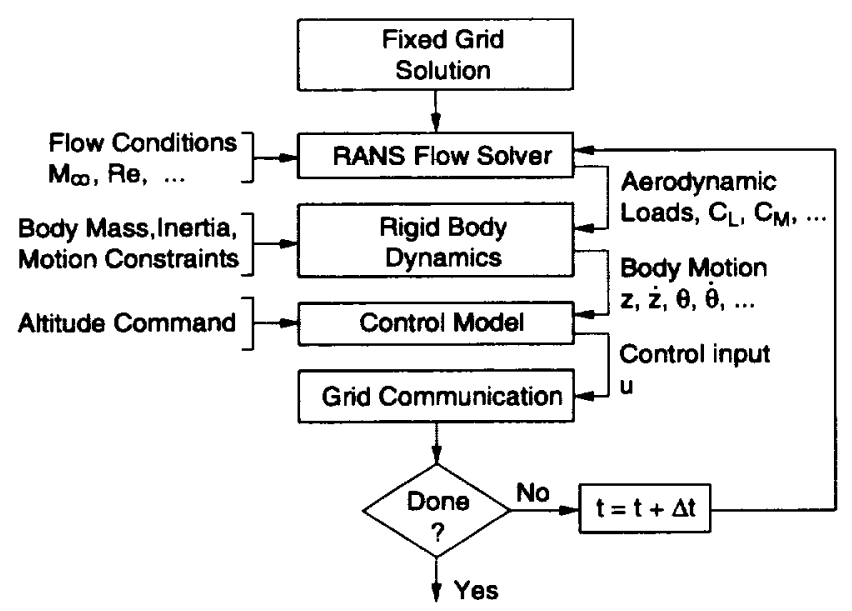

FIG. 1. Ovenall coupled system appraach.

trajectory of a descending powered-lift aircraft [2], and the coupled fluids/body dynamics problem [3]. The coupled fluid, dynamics, and controls problem has been previously investigated for a three-dimensional store separation problem [4]. The case demonstrated here uses a similar methodology to describe the fluid and body dynamics by using a diagonalized implementation of the RANS equations in an overset mesh framework and Euler's equations of motion to describe the rigid body response to aerodynamic loads. This study is different from the store separation problem as the interaction between fluid dynamics, rigid body motion, and controls, for the store problem was small. This was a result of the large ejection force on the missile, which dominated the trajectory of the store. Here, the interaction between fluids, rigid body dynamics, and controls is much more complex. The problem explored here will demonstrate the potential of coupling these disciplines for prototyping control systems in nonlinear flight regimes.

2. Approach. The coupling used to solve this problem is shown schematically in Fig. 1. This simulation starts with a converged fixed grid solution. The integrated aerodynamic loads and body states are passed from the flow solver to the six degrees-of-freedom, rigid body dynamics code. The new body position is then integrated one time step given the mass, moment-of-inertia, and applied forces. Any kinematic constraints on the body are also applied to the body motion at this time. The new body state is then passed to the controls module which generates a control input given the commanded altitude. Finally, the overlapped airfoil and jet grids are repositioned on the background mesh. This repositioning of the grids requires that the intergrid communication between the overlapped grids be re-established. This entire process is repeated every time step until the simulation is complete.

2.1. Flow Solver. The flow field is computed by solving the RANS equations, using the diagonal scheme of Pulliam and Chaussee [5] in the grid framework of Steger et al [6]. The equations are integrated through Euler implicit time marching and second-order spatial differencing with viscous wall conditions specified as no-slip, zero normal pressure gradient, and adiabatic. Information transfered between overset mesh boundaries was implemented using trilinear interpolation of the dependent variable vector, $\mathbf{Q}=[\rho, \rho u, \rho v, \rho w, e]^{T}$. The flow solver cost is $7 \mu s /$ cell/step using a single processor of a Cray C90. Turbulence was modeled using the algebraic model of Baldwin and Lomax [7], as implemented by Renze [8].

2.2. Domain Decomposition. Computation of the loads generated by a dynamic vehicle requires accurate representation of the geometry and flow field. For the cases depicted in Fig. 2 and 3, the domain 
was discretized using a curvilinear airfoil and nozzle grids overset on a stretched Cartesian background mesh. Overlapped grid topologies of this type allow representation of complex geometries and movement of bodies without regeneration of component grids $[2,4,9]$. Since grid boundaries are not required to match neighboring grids, robust algebraic [10] and hyperbolic [11] grid generators can be used to obtain spacing and orthogonality control.

The exchange of flow information between the zones of an overlapping grid system are computed using a domain connectivity function. The donor-receiver relationship between the grids was established at each time step using an efficient search technique [12]. The relative cost of the intergrid communication to flow solver expense is dependent on the ratio of intergrid boundary points to volume points.

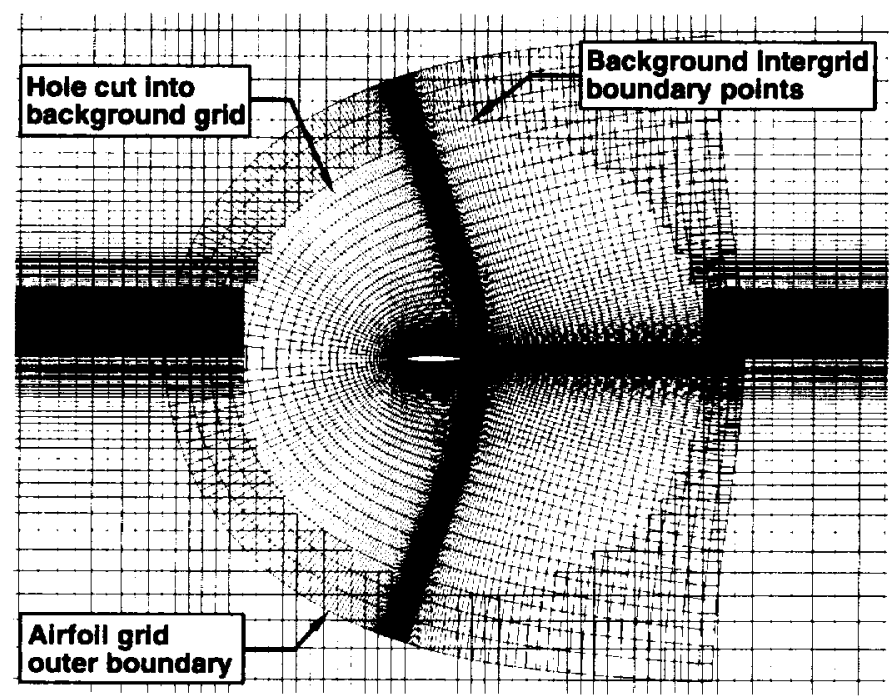

FIG. 2. A closeup view of the airfoil grid overset on a Cartesian backgnound grid.

The overset mesh topology used for the airfoil with jets is shown in Fig. 2 and 3. Here, the intergrid boundaries are comprised of the outer boundary of the airfoil and nozzle grids, as well as the hole boundary in the background and airfoil grids. Along the hole boundary in the background grid, $\mathbf{Q}$ is trilinearly interpolated from the solution field of the airfoil grid. Similarly, the outer boundary points of the airfoil grid are interpolated from the dependent variable vector of the background grid. This exchange of information establishes the intergrid communication between the grid zones for steady or unsteady fixed grid problems. Note that the boundary surface points of each implicit grid zone must be specified either by boundary conditions or through communication with other zones. For problems in which zones move relative to each other, as is the case for aerodynamically controlled vehicles, the intergrid communication must be re-established at each time step.

Note that Fig. 3 shows the bottom nozzle grid at the trailing edge of the airfoil with an outline of the top nozzle grid. The upper part of the bottom nozzle grid, which extends above the airfoil surface, is fictitious and does not interact with the flow field. These nozzle grids are used to allow the flow to develop as the mass flow rate is changed by the active controller at the inflow boundary.

2.3. Rigid Body Dynamics. Rigid body motion is described by a conservation of linear and angular momentum using coordinates fixed to the center of gravity of the body and aligned with its principal axes [3]. Euler parameters are used to describe the rotation of the body from its initial position. These parameters are integrated according to the rotational body dynamics, updated, and stored for each grid [12, 13]. Kinematic 




FIG. 3. The bottom nozzle grid at the trailing edge of the airfoil with an outline of the top nozzle grid.

constraints can be imposed during the trajectory for restricted degrees-of-freedom simulations. In addition, the assumption of rigid body dynamics eliminates the need to store the component grids for all time, inasmuch as the Euler parameters may be used to compute grid attitude from the initial position. Note that the time scales of fluid motion are typically many orders smaller than the rigid body motion, making this loose coupling between the body motion and flow solver adequate.

2.4. Control Law. The airfoil controlled by an applied pitching moment used an optimal state feedback control law. Integral control was used to improve tracking performance of altitude commands. The altitude of the airfoil was controlled by adding an applied moment to the aerodynamic forces, changing the attitude of the airfoil. This change in attitude produces aerodynamic lift, accelerating the airfoil vertically. The controller was designed using a linear time invariant system, based on quasi-steady aerodynamic theory. The feedback gains were then calculated using a Linear Quadratic Regulator (LQR) method, resulting in optimal feedback gains for the linearized model.

In the second problem, the applied moment was replaced by two reaction control jets near the trailing edge. Due to the nonlinear nature of this system, a robust control design approach was used [14]. The linear plant model used in the control design was developed using input/output data generated by the coupled system. By using robust control design methods, a nominal linear model of the airfoil system can be parameterized by a measure of uncertainty. This parameterized uncertainty introduces unmodeled dynamics not accounted for by the nominal model. Therefore, by increasing the uncertainty in the model, the control design becomes increasingly robust to unmodeled dynamics not present in the linear nominal model.

3. Results. The coupled system methodology described above was applied to a two-dimensional airfoil with and without jets. Both systems had controllers designed to track altitude commands. The closed-loop response of the airfoil without jets, using the coupled system, was compared to a quasi-steady aerodynamic model. The tracking performance of the airfoil with jets was evaluated from a closed-loop simulation of the coupled system.

3.1. Airfoil Controlled by Applied Moment. This system consists of a NACA 64A010 airfoil with an applied pitching moment added to the aerodynamic forces. The airfoil has two degrees-of-freedom, one in 


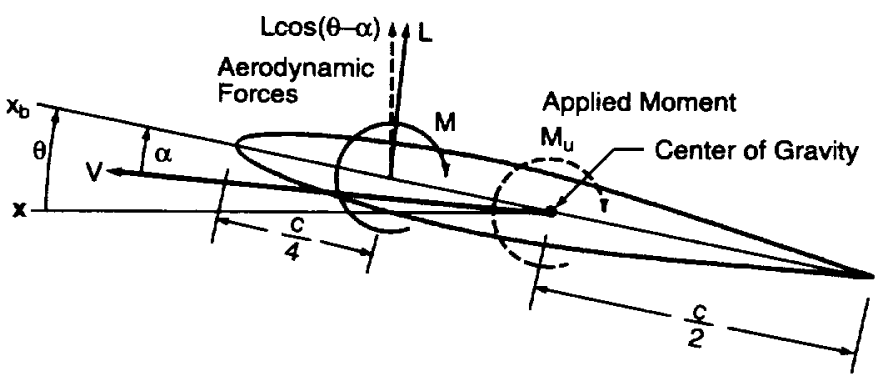

Fig. 4. Aerodynamic forces and notation.

the vertical direction and the other in the pitch direction. A comparison of the closed-loop response of the coupled system was made by replacing the RANS equations with a quasi-steady aerodynamic model. This comparison will provide some measure of validation for the coupled system.

3.1.1. Aerodynamic Model. The aerodynamic lift and moment coefficients for the airfoil are modeled using a quasi-steady formulation [15]. These coefficients are expressed as

$$
\begin{aligned}
& C_{L}(t)=C_{L_{o}}+C_{L_{\alpha}} \alpha(t)+C_{L_{\dot{\alpha}}} \frac{l}{V} \dot{\alpha}(t)+C_{L_{q}} \frac{l}{V} q(t) \\
& C_{M}(t)=C_{M_{o}}+C_{M_{\alpha}} \alpha(t)+C_{M_{\dot{\alpha}}} \frac{l}{V} \dot{\alpha}(t)+C_{M_{q}} \frac{l}{V} q(t)
\end{aligned}
$$

where $C_{L}$ and $C_{M}$ are the coefficients of lift and pitching moment about the quarter chord location respectively. The aerodynamic lift and moment in terms of $C_{L}$ and $C_{M}$ were

$$
L(t)=C_{L}(t)\left(\frac{1}{2} \rho V^{2} S\right), \quad M(t)=C_{M}(t)\left(\frac{1}{2} \rho V^{2} S c\right)
$$

The stability derivatives $C_{L_{\alpha}}, C_{L_{q}}, C_{M_{\alpha}}$, and $C_{M_{q}}$ were evaluated from steady state solutions of the RANS equations. The $C_{L_{\dot{\alpha}}}$ and $C_{M_{\alpha}}$ stability derivatives were estimated from unsteady flow solutions of a plunging airfoil at a reduced frequency of $k=0.7$. The terms, $C_{L_{o}}$ and $C_{M_{o}}$ represent the lift and moment coefficients evaluated at $\alpha=\dot{\alpha}=q=0$. All stability derivatives were evaluated for a free stream Mach number of 0.3 , Reynolds number of $32 \cdot 10^{6}$, and a chord length of $4.6 \mathrm{~m}$. The values calculated for the quasi-steady model are given in Table 1.

TABLE 1

Quasi-Steady Coefficients

\begin{tabular}{|cc|cc|}
\hline$C_{L_{0}}$ & 0.00 & $C_{M_{0}}$ & 0.00 \\
$C_{L_{\alpha}}$ & 6.28 & $C_{M_{\alpha}}$ & 0.00 \\
$C_{L_{q}}$ & 1.44 & $C_{M_{q}}$ & -0.36 \\
$C_{L_{\dot{\alpha}}}$ & 0.78 & $C_{M_{\dot{\alpha}}}$ & -0.35 \\
\hline
\end{tabular}

3.1.2. Linearized Dynamic Model. The equation of motion used for the vertical direction, $m \ddot{z}=$ $\sum F_{z}$, can be expressed as

$$
m \ddot{z}(t)=L(t) \cos (\theta(t)-\alpha(t))
$$

where gravitational and drag forces are neglected. Since the lift, $L(t)$ is perpendicular to the total velocity of the airfoil, as shown in Fig. 4, it was multiplied by $\cos (\theta-\alpha)$ to obtain its vertical component. 




FIG. 5. A block diagram of the closed-loop system.

The equation of motion in the pitch direction, $I_{y y} \ddot{\theta}=\sum M_{y}$, can be expressed as

$$
I_{y y} \ddot{\theta}(t)=M(t)+L(t) \cos (\alpha(t)) \frac{c}{4}+M_{u}(t)
$$

where $M_{u}$ was the applied pitching moment produced by the controller.

The quasi-steady aerodynamic lift and moment equations were substituted into Eq. (1) and (2) and linearized about the zero state. These equations were expressed as the linear time invariant system

$$
\dot{\mathbf{x}}_{1}(t)=A_{1} \mathbf{x}_{1}(t)+B_{1} u(t) .
$$

where $\mathbf{x}_{1}=[\theta, \dot{\theta}, z, \dot{z}]^{T}$. Numerical values for $A_{1}$ and $B_{1}$ were

$$
A_{1}=\left[\begin{array}{cccc}
0 & 1 & 0 & 0 \\
3380 & 2.37 & 0 & -33.2 \\
0 & 0 & 0 & 1 \\
7780 & 123 & 0 & -76.2
\end{array}\right], B_{1}=\left[\begin{array}{c}
0 \\
2580 \\
0 \\
0
\end{array}\right]
$$

These matrices were calculated using $m=62.8 \mathrm{~kg}, I_{y y}=386 \mathrm{~kg} \cdot \mathrm{m}^{2}, M_{\infty}=0.3$, and the coefficients in Table 1.

3.1.3. State Feedback Design. The state feedback controller used in this study, incorporates integral control to improve tracking performance. Figure 5 shows a block diagram of the state feedback controller used. The objective of this control design is to find the optimal state feedback gains such that the altitude, $z(t)$, tracks a commanded altitude path, $z_{c}(t)$. Therefore, the error was defined as

$$
e(t)=z(t)-z_{c}(t)=D \mathbf{x}_{\mathbf{1}}(t)-z_{c}(t)
$$

Using state and integral feedback, the input $u$ was

$$
u(t)=G_{1} \mathbf{x}_{1}(t)+G_{2} \int e(t) d t
$$

where $G_{1}$ and $G_{2}$ are the constant feedback gains. Now let, $\dot{x}_{2}(t)=e(t)$, where

$$
\dot{x}_{2}(t)=D \mathbf{x}_{1}(t)-z_{c}(t)
$$

Combining the linear systems from Eqs. (3) and (4) results in

$$
\left[\begin{array}{c}
\dot{\mathbf{x}}_{1} \\
\dot{x}_{2}
\end{array}\right]=\left[\begin{array}{cc}
A_{1} & 0 \\
D & 0
\end{array}\right]\left[\begin{array}{l}
\mathbf{x}_{1} \\
x_{2}
\end{array}\right]+\left[\begin{array}{c}
B_{1} \\
0
\end{array}\right] u+\left[\begin{array}{c}
0 \\
-I
\end{array}\right] z_{c}
$$


This can be expressed as one linear system

$$
\dot{\mathbf{x}}(t)=A \mathbf{x}(t)+B u(t)+F z_{c}(t)
$$

where $\mathbf{x}(t)=\left[\begin{array}{ll}\mathbf{x}_{1}(t) & x_{2}(t)\end{array}\right]^{T}$. The input $u(t)$ for state feedback becomes

$$
u(t)=\left[\begin{array}{l}
G_{1} \\
G_{2}
\end{array}\right]\left[\begin{array}{l}
\mathbf{x}_{1}(t) \\
x_{2}(t)
\end{array}\right]=G \mathbf{x}(t)
$$

Optimal feedback gains were calculated by finding the input, $u(t)$, which minimizes the quadratic integral cost function

$$
J(u(t))=\int_{0}^{\infty}\left(\mathbf{x}^{T}(t) Q \mathbf{x}(t)+u^{T}(t) R u(t)\right) d t
$$

where $Q$ and $R$ are positive-semidefinite symmetric matrices. The quantity, $\mathbf{x}^{T}(t) Q \mathbf{x}(t)$, is a measure of how far the states deviate from the zero state at time $t$. The matrix $Q$ is a diagonal weighting matrix, where each diagonal element corresponds to a penalty on one of the states. The term, $u^{T}(t) R u(t)$, accounts for the amplitude of the input used to bring the system to the zero state. This optimal control design is known as the LQR problem.

The solution to this optimization problem is well known [16] and it can be shown that the input which minimizes $J(u)$, given the system $\dot{\mathbf{x}}(t)=A \mathbf{x}(t)+B u(t)$ and the initial condition $x(0)=0$, is

$$
u_{o p t}(t)=-R^{-1} B^{T} X \mathbf{x}(t)
$$

Here, $\mathrm{X}$ is the solution to the algebraic Riccati equation

$$
A^{T} X+X A-X B R^{-1} B^{T} X+Q=0
$$

where $A-B R^{-1} B^{T} X$ is stable. Thus, the optimal state feedback gains are

$$
G=-R^{-1} B^{T} X
$$

The optimal feedback gains $G$ were calculated by first choosing initial values for the weights $Q$ and $R$. After calculating the feedback gains $G$, the closed-loop response was simulated by solving Eqs. (1) and (2) coupled with the quasi-steady aerodynamic model. These coupled equations were integrated in time using a fourth order Runge-Kutta scheme. To assess the performance of the controller, a step in the commanded altitude of one chord length was simulated. The desired transient response for this simulation was a settling time of $1.0 \mathrm{~s}$ with minimum overshoot. If this performance criteria was not achieved, the weights were adjusted to increase or decrease the penalty of the individual states. This change in the weights resulted in a optimal controller with a new transient response. This cycle was iterated upon until the desired performance was achieved. The final iteration produced the feedback gains

$$
G=\left[\begin{array}{lllll}
-193 & -13.1 & -2.43 & 1.07 & -3.00
\end{array}\right] \cdot 10^{3}
$$

3.1.4. Airfoil with Applied Moment Simulation. A step command in altitude of one chord length from the starting position was simulated. Figure 6 shows states of the controlled simulation for the linear aerodynamic model and the nonlinear coupled system response. These two simulations match up very well as would be expected in this linear flight regime. This comparison provides a measure of validation for the 

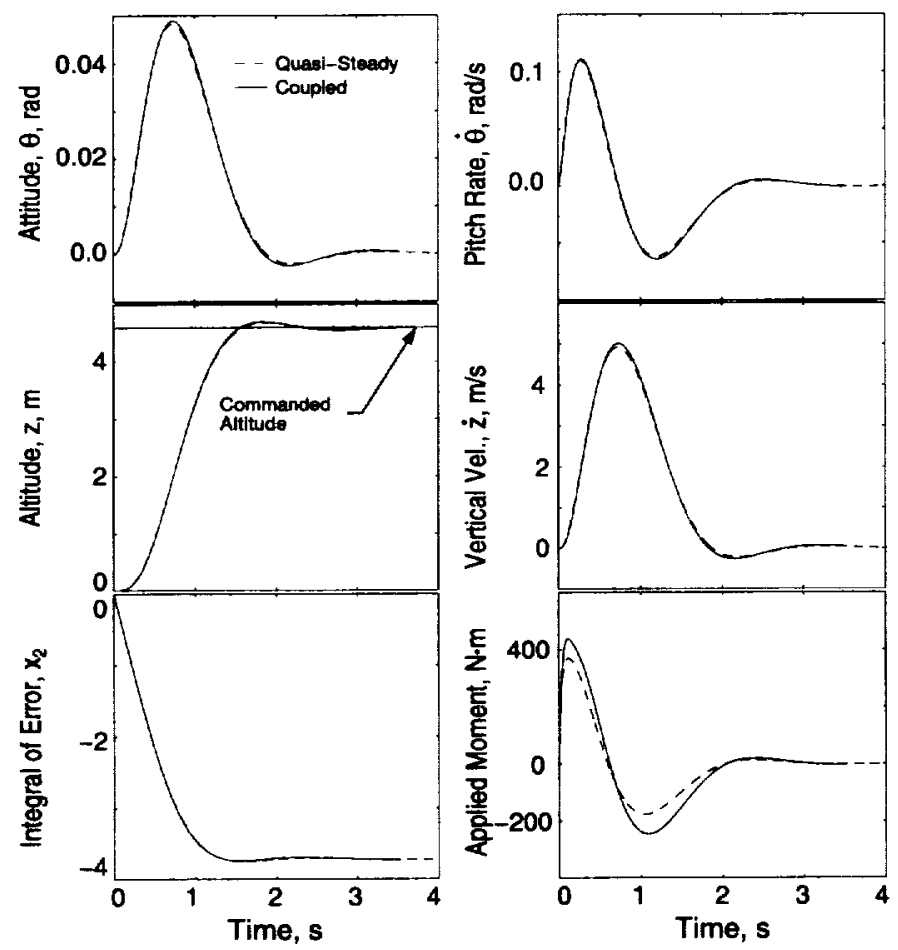

FiG. 6. Controlled response of the linear and coupled system to a step input of $4.6 \mathrm{~m}$ in the commanded altitude.

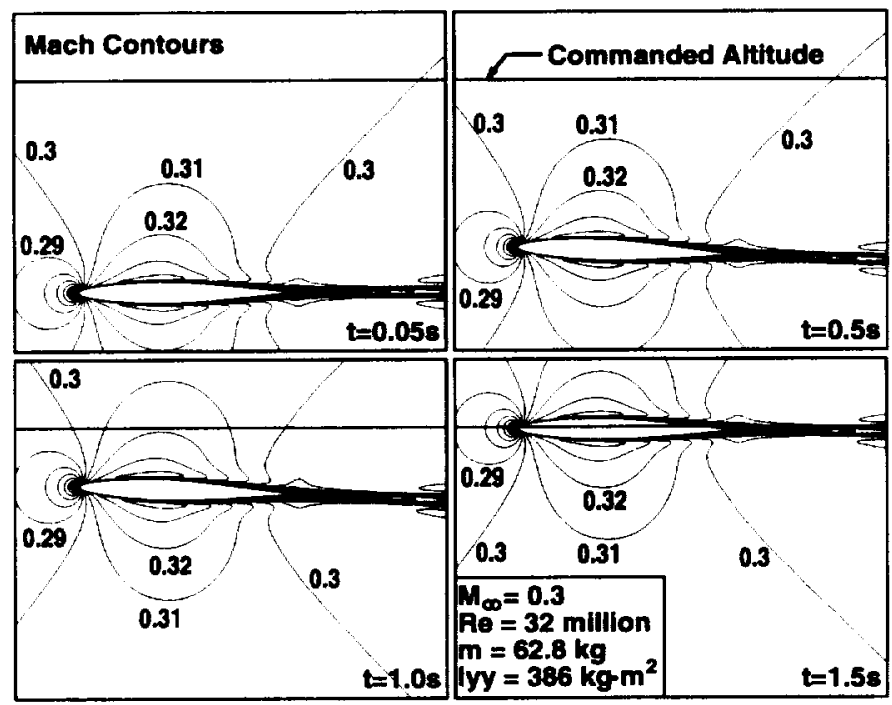

FIG. 7. Instantaneous Mach contours during the ascent of the airfoil to the commanded altitude.

nonlinear coupled system. Instantaneous Mach contours of the simulation for the ascent of the airfoil to the reference altitude, is shown in Fig. 7 at four different times.

In this simulation, the applied moment pitches the airfoil up producing aerodynamic lift. This lift accelerates the airfoil in the vertical direction, moving the airfoil to the commanded altitude. As the airfoil starts to reach the commanded altitude, the controller produces a downward pitching moment. This applied moment pitches the airfoil down which reduces the lift generated by the airfoil, slowing down its ascent. Figure 6 shows a small amount of overshoot of the commanded altitude, as predicted in the control design 
using the quasi-steady model. This overshoot was also seen in the RANS coupled simulation. A slight difference in the control moment generated can be seen in Fig. 6 where the quasi-steady model predicts a smaller control input.

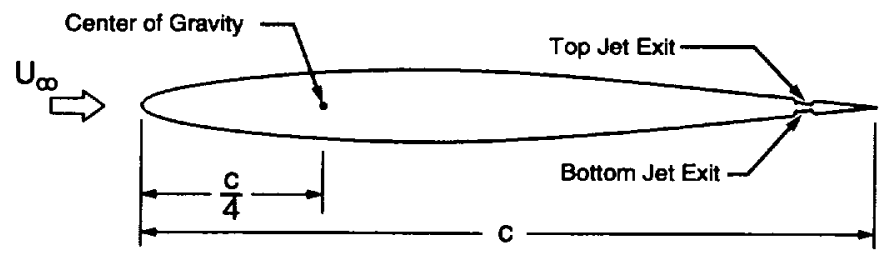

FIG. 8. Two-dimensional airfoil with jets problem.

3.2. Airfoil Controlled by Jets. In this problem, the applied moment is replaced by two reaction control jets near the trailing edge of the airfoil. The center of gravity is placed at the quarter chord location as shown in Fig. 8, increasing the stability of the system.

It should be noted that the problem of resolving the complex flow of a two-dimensional jet in a cross flow was not addressed here, since the purpose of this investigation was to demonstrate the analysis of a control system in a nonlinear flight regime. Therefore, an approximation to the jet flow was made by using coarse grids which do not resolve the complex flow associated with jets. However, it maybe argued that the approximation made here is satisfactory for control design and analysis purposes as long as the flow being resolved captures the general dynamics of the system.

3.2.1. Plant Model Design. The highly nonlinear aerodynamic effects, from the jets at the trailing edge of the airfoil, make it very difficult to model. Figure 9 shows the aerodynamic lift and moment time histories for a fixed airfoil with a constant mass flow rate from the bottom jet. The aerodynamic forces have a large oscillatory behavior produced by the unsteady flow generated behind the jet. The large magnitude of these oscillations were due to the jet being two-dimensional. The power spectral density of the $C_{L}$ time history from $t U_{\infty} / c=0$ to 10 is shown in Fig. 10. This figure shows that the lift generated by a constant mass flow rate to the bottom jet does not have a dominate frequency. The approach used to model the dynamic response of the airfoil was to perform a system identification using input/output data. This input/output data was generated from two open-loop simulations using the coupled system. Note that a turbulence model was not used for this problem as it was not valid for this complicated unsteady flow field.

The relationship between the input $u$ and nondimensional mass flow rate to the top and bottom jets is shown in Fig. 11. A positive input $u$ would correspond to a mass flow rate to the bottom jet and a negative $u$ for the top jet, where $u$ has a magnitude limit of 0.3 .

The variables $u_{T}$ and $u_{B}$ are functions of the input $u$ and are defined in the following way:

$$
u_{T}=\left\{\begin{array}{cc}
0 & u \leq 0 \\
u & 0<u \leq 0.3 \\
0.3 & 0.3<u
\end{array} \quad u_{B}=\left\{\begin{array}{cc}
0.3 & u<-0.3 \\
-u & -0.3 \leq u<0 \\
0 & 0 \leq u
\end{array}\right.\right.
$$

A delay for the mass flow rate command and the actual mass flow rate applied to the nozzles is modeled by $1 /(\tau s+1)$, as shown in Fig. 11, where $\tau=4.5 \cdot 10^{-4}$.

The nondimensional mass flow rates, $\dot{m}_{t o p}^{*}$ and $\dot{m}_{b o t}^{*}$ were applied to the top and bottom nozzle grids respectively. These mass flow rates were used to change the inflow boundary conditions on the nozzle grids shown in Fig. 3. Note that a value of $\dot{m}^{*}=0.1$ would corresponds to a mass flow rate of $\dot{m}=13.7 \mathrm{~kg} / \mathrm{s}$ at the inflow boundary. 


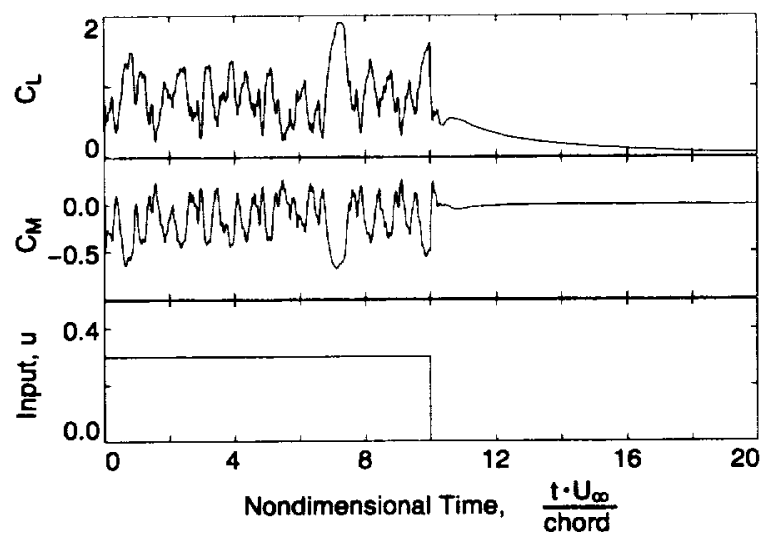

FIG. 9. Time history of $C_{L}$ and $C_{M}$ for a fixed airfoil unth the bottom jet turned on for a nondimensional mass flow rate of 0.3 from $t U_{\infty} / c=0$ to 10 . Time is nondimensionalized in terms of chord distances flown.

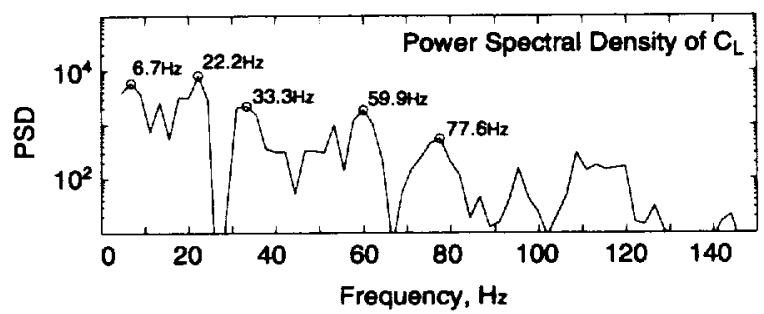

FIG. 10. The power spectral density of the $C_{L}$ time history shoun in Fig. 9 from $t U_{\infty} / c=0$ to 10, where $U_{\infty}=102 \mathrm{~m} / \mathrm{s}$ and $c=4.6 \mathrm{~m}$.

The state space model developed has an input $u$, with outputs $\dot{z}$ and $\theta$. The altitude $z$ was found by integrating $\dot{z}$. The pitch rate was produced by restructuring the linear system to obtain $\dot{\theta}$. This was done by first looking at the output equation

$$
\mathbf{y}=\left[\begin{array}{c}
\dot{z} \\
\theta
\end{array}\right]=\left[\begin{array}{c}
c_{1} \\
c_{2}
\end{array}\right] \mathbf{x}
$$

Thus the pitch rate could be expressed as

$$
\dot{\theta}=c_{2} \dot{\mathbf{x}}=c_{2}(A \mathbf{x}+B u)
$$

Restructuring the output equation to include $\dot{\theta}$ resulted in

$$
\mathbf{y}=\left[\begin{array}{c}
\dot{z} \\
\boldsymbol{\theta} \\
\dot{\theta}
\end{array}\right]=\left[\begin{array}{c}
c_{1} \\
c_{2} \\
c_{2} A
\end{array}\right] \mathbf{x}+\left[\begin{array}{c}
0 \\
0 \\
B
\end{array}\right] u
$$

This system, $P(s)$, is shown in Fig. 12 where the altitude was found by integrating the vertical velocity.

The input $u$ and states $\mathbf{x}$, for the open-loop simulations, are shown in Fig. 13 where $u(t)$ was a series of step commands. The first simulation is shown in Fig. 13a and the second in Fig. 13b. The model was identified using both sets of input/output data simultaneously. A comparison of the coupled simulation and linear model is shown in Fig. 13. This comparison shows how the simple two state model captures the general behavior of the coupled system for the given inputs. The model compares relatively well for 


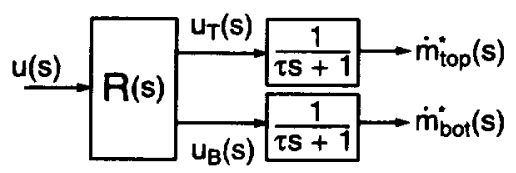

FIG. 11. Block diagram shouing the relation between the input $u$ and the nondimensional mass flow rates to the top and bottom jets in the Laplacian domain.

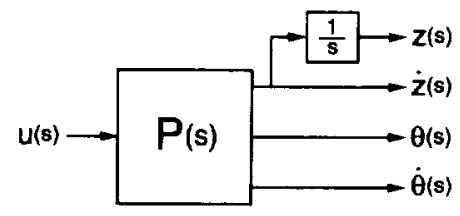

FIG. 12. State space model with integrator and added $\dot{\theta}$ output.

the second simulation and for the beginning of the first simulation. In the first simulation, the model and coupled response start to diverge near $t=0.1 \mathrm{~s}$. This could be a result of the larger attitude and pitch rate as compared to the second simulation.

3.2.2. Robust Control Design. The model used for the robust control design consists of the nominal model $P(s)$ and the elements, $\gamma$ and $\Delta$, which parameterize the uncertainty in the model. This system is shown in Fig. 14 where $\gamma$ was a scalar and determines the amount of uncertainty in the model. The transfer function $\Delta$ is assumed stable and unknown, except for the norm condition, $\|\Delta\|_{\infty} \leq 1$. The interconnection for the inputs $w$ and outputs $v$ to the $\Delta$ block are shown in Fig. 14a. The perturbed system with $\Delta$ connected is shown in Fig. 14b.

The block diagram in Fig. 15 shows the performance weights and disturbances used for the robust control design [14]. The $W_{\text {model }}$ block was the ideal model response, which was represented by a well damped secondordered system. The performance weight $W_{c m d}$ defines the magnitude and frequency of the input commands to be tracked. $W_{a c t 1}$ and $W_{a c t 2}$ were penalty weights on the input $u$ and its rate $\dot{u}$. The difference in the altitude and the ideal altitude response was weighted by $W_{\text {perf } 1 \text {. The attitude, pitch rate, and vertical }}$ velocity were weighted by $W_{\text {perf } 2}$. Noise was added to the system by inputs $d_{3}$ to $d_{5}$ and shaped by $W_{\text {snois }}$ which sets the magnitude and frequency range.

The performance weights and plant $G$ in Fig. 15 were reduced to the block $M$ shown in Fig. 16. The controller $K$ was designed so that the perturbed system was stable and the transfer function from $\mathbf{d}$ to $\mathbf{e}$ satisfies

$$
\left\|T_{e d}(M, K, \Delta)\right\|_{\infty}<1
$$

for all stable perturbations, $\Delta$ satisfying $\|\Delta\|_{\infty} \leq 1$.

The controller $K$ was determined by using $\mu$ synthesis, which was approximated by an iterative method known as " $D-K$ iterations" [14]. The original controller $K$, which had 68 states, was reduced to 17 states by performing a balanced realization [17]. This reduced controller was then used for the coupled RANS simulation.

3.2.3. Airfoil with Jets Simulation. The input $\mathbf{y}$ to the controller $K$ was a vector containing the commanded altitude and the states, where $\mathbf{y}=\left[z_{c}, z, \dot{z}, \theta, \dot{\theta}\right]$. The controller takes these inputs and generates the signal $u(t)$, which was passed through the block shown in Fig. 11. This produces mass flow rates for the inflow boundary on the nozzle grids. 

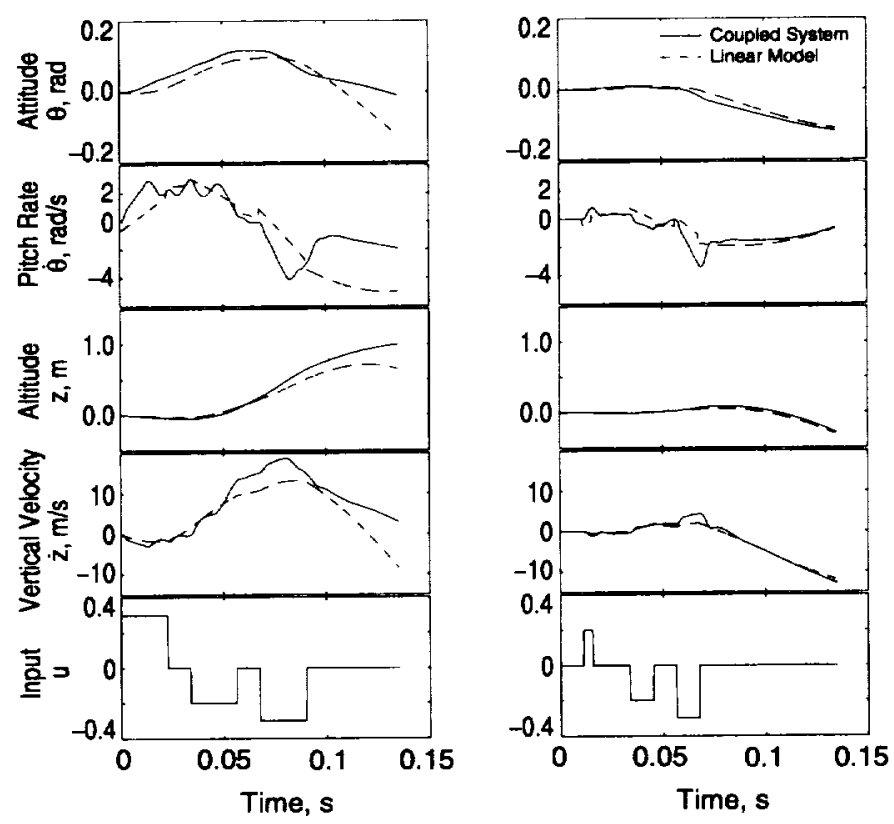

a) Case 1

b) Case 2

FIG. 13. Two open-loop simulations of the nonlinear coupled system with the linear input/output model response.
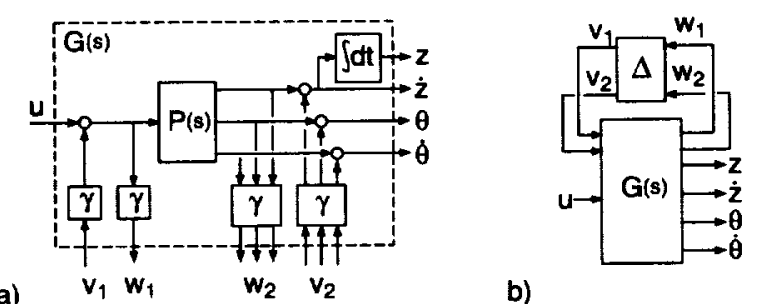

b)

FIG. 14. Uncertainty model for $P(s)$. a) Uncertainty block $\Delta$ not connected where $\gamma$ determines the amount of uncertainty; b) Plant $G(s)$ with full uncertainty block $\Delta$.

Performance of the controller was evaluated by analyzing the closed-loop response of the airfoil to a step input in the commanded altitude. Figure 17 shows the states for the simulations using the the linear and nonlinear models. The altitude trajectory for the linear model was very good and was nearly identical to the ideal model response. The altitude trajectory for the coupled system was also good. It showed that the controller was able to track the desired altitude trajectory within the prescribed tracking performance.

The nonlinear simulation also revealed oscillations in the control jets not shown in the linear model simulation. The difference between these two simulations show how the nonlinear dynamics were not captured by the linear plant model. These oscillations are induced by the controller, which is trying to force the nonlinear airfoil system follow the desired altitude response of a linear system model, $W_{\text {model }}$. The frequency of these oscillations are thought to be partly related to the altitude tracking performance, $W_{\text {perf } 1}$, used to design the controller. By increasing the tracking performance, the controller will try to keep the airfoil even closer to the desired altitude path, increasing the frequency of the oscillations.

The instantaneous Mach contours for the coupled simulation are shown in Fig. 18 at four different times. This figure reveals the complicated flow pattern generated by the top and bottom jets during the closed-loop simulation. The snapshot at $t=0.02 s$ was taken at the beginning of the simulation where the top jet was 


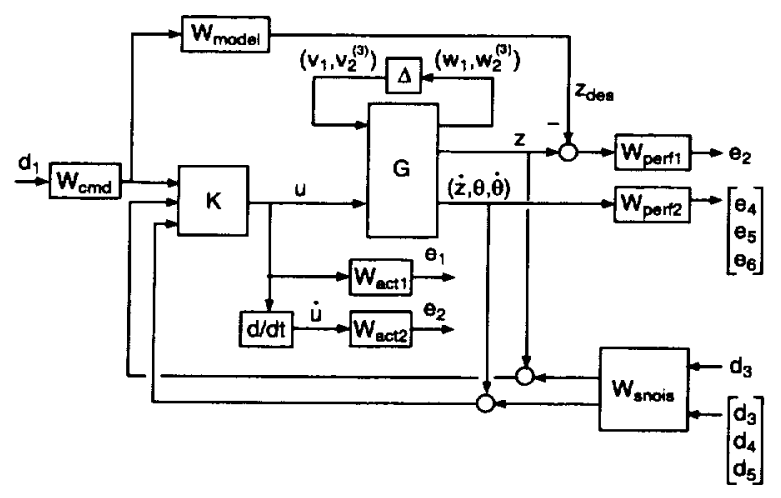

FIG. 15. Block diagram of performance weights for robust control design.

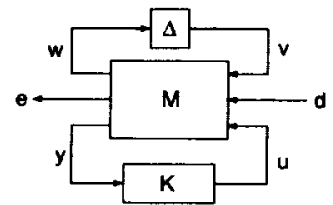

FIG. 16. Closed-loop system with performance weights and plant model $G$ contained in $M$.

turned on by the controller. This top jet produces aerodynamic forces pitching the airfoil up. As the airfoil pitches up, aerodynamic lift is generated and begins to move the airfoil vertically. The instantaneous Mach contours at $t=0.4 \mathrm{~s}$ show the unsteady wake behind the airfoil as a result of the oscillations in the top and bottom jets. A closeup view of the trailing edge can be seen in the last two snapshots of Fig. 18. This view shows the unsteady flow behind the top jet where a low pressure region has developed. At $t=1.2 \mathrm{~s}$ the airfoil has reached the commanded altitude.

4. Conclusions. The analysis of an aircraft controls system for nonlinear flight regimes is complicated by the interaction of fluid dynamics, rigid body dynamics, and the control system itself. Of particular interest is the landing of a powered-lift aircraft which has a strong interaction between the lifting jets and ground plane. Towards this goal of analyzing this system, the coupling of the Reynolds-averaged Navier-Stokes equations and Euler's equations of rigid body motion with an active control system, was performed. As a demonstration problem, the altitude control of an airfoil with and without jets was investigated.

The airfoil without jets was controlled by an applied moment and used an optimal state feedback controller with integral control. The control design used a Linear Quadratic Regulator method with a linear time invariant system based on quasi-steady aerodynamics. The stability derivatives for this model were computed using steady and unsteady solutions to the RANS equations. A step in the commanded altitude was simulated by the RANS coupled system which compared well with the quasi-steady model. This comparison provided a measure of validation for the nonlinear coupled system.

The airfoil controlled by jets used a robust control design method. The linear plant model, used in the control design, was developed by performing a system identification on the input/output data. This input/output data was generated by two open-loop simulations of the coupled system. Simulation of the closed-loop response of the airfoil with the coupled system, demonstrated that the airfoil was able to track the desired altitude trajectory within the specified performance. It also showed oscillations in the control jets that were not predicted by the linear model simulation. 

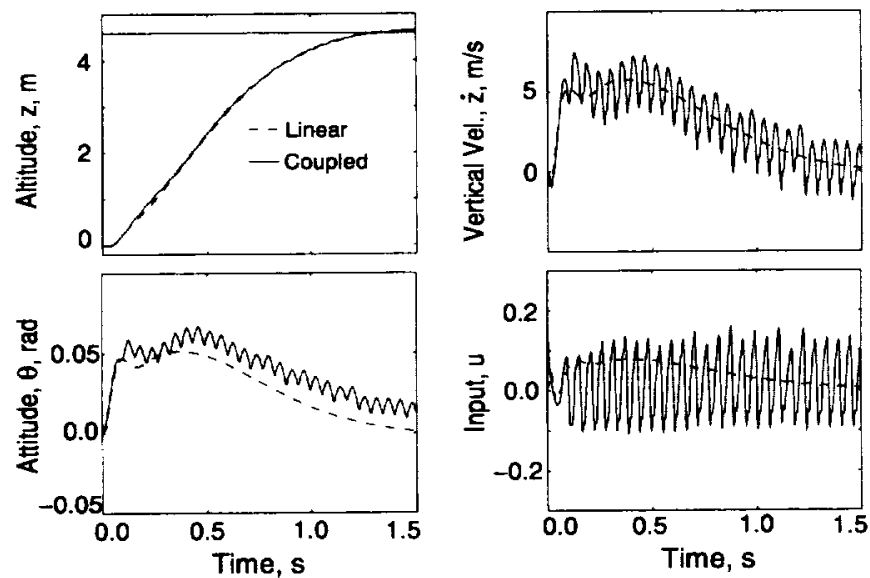

FIG. 17. A comparison of the states for the linear and coupled simulation.

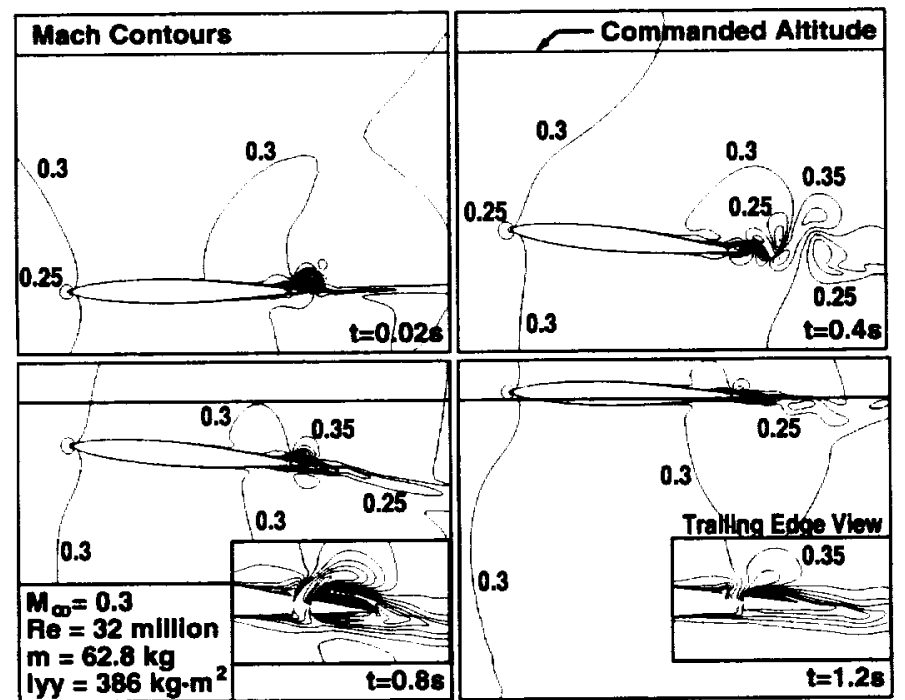

FIG. 18. Instantaneous Mach contours during the ascent of the airfoil to the commanded altitude.

\section{REFERENCES}

[1] C. A. Atwood AND W. R. VAN DALSEM, Flowfield simulation about the SOFIA airborne observatory, AIAA J. of Aircraft, 30 (1993), pp. 719-727.

[2] K. Chawla AND W. R. VAN DALSEM, Numerical simulation of STOL operations using thrustvectoring, AIAA Paper 92-4254, 1992.

[3] R. L. MEAKIN AND N. SUHS, Unsteady aerodynamic simulation of multiple bodies in relative motion, AIAA Paper 89-1996, 1989.

[4] C. A. ATwood, Computation of a controlled store separation from a cavity, AIAA J. of Aircraft, 32 (1995), pp. 846-852.

[5] T. H Pulliam AND D. S. Chaussee, $A$ diagonal form of an implicit approximate-factorization algorithm, J. Comput. Phys. 39 (1981), pp. 347-363.

[6] J. L. Steger, F. C. Dougherty, And J. A. Benek, A Chimera Grid Scheme, in Advances in Grid 
Generation, K. N. Ghia and U. Ghia, eds., American Society of Mechanical Engineers, New York, 1993, pp. 59-69.

[7] B. S. BALDWIN AND H. LOMAX, Thin-layer approximation and algebraic model for separated turbulent flows, AIAA Paper 78-257, 1978.

[8] K. RENZE, P. Buning, AND R. RAJAgoplan, A comparative study of turbulence models for overset grids, AIAA Paper 92-0437, 1992.

[9] R. L MEAKIN, Moving body overset grid methods for complete aircraft tiltrotor simulations, AIAA Paper 93-3350, 1993.

[10] J. P. Steinbrenner, J. R. Chawner, and C. L. Fouts, A structured approach to interactive multiple block grid generation, in Advisory Group for Aerospace Research \& Development, Conference Proceedings No. 464, Applications of Mesh Generation to complex 3-D Configurations, 1989, pp. 8.1-8.12.

[11] W. M. ChAN AND J. L. STEgER, Enhancements of a three-dimensional hyperbolic grid generation scheme, Appl. Math. and Comput. 51 (1992), pp. 181-205.

[12] R. L MEAKIN, A new method for establishing inter-grid communication among systems of overset grids, AIAA Paper 91-1586, 1991.

[13] R. L MEAKIN, Computations of the unsteady flow about a generic wing/pylon/finned-store configuration, AIAA Paper 92-4568, 1992.

[14] A. Packard, J. Doyle, And G. Balas, Linear, multivariable robust control with a $\mu$ perspective, ASME J. of Dynamic Systems, Measurement, and Control 155 (1993), pp. 426-437.

[15] M. Tobak AND L. B. SchifF, Aerodynamic mathematical modeling - basic concepts, in Advisory Group for Aerospace Research \& Development, Lecture Series No. 114, Dynamics Stability Parameters, 1981, pp. 1.1-1.32.

[16] H. KWAkernaAK And R. Sivan, Linear Optimal Control Systems, John Wiley \& Sons, New York, 1972.

[17] K. GLOVER, All optimal hankel-norm approximations of linear multivariable systems and their $L_{\infty}$ error bounds, International J. of Control, 39 (1984), pp. 1115-1193. 
Public reporting burden for this collection of information is estimated to average 1 hour per response, including the time for reviewing instructions, searching existing data sources, gathering and maintaining the data needed, and completing and reviewing the collection of information. Send comments regarding this burden estimate or any other aspect of this Davis Highway, Suite 1204, Arlington. VA 22202-4302, and to the Office of Management and Budget. Paperwork Reduction Project (07040188). Washington, DC 20503.

\begin{tabular}{l|l|l}
\hline 1. AGENCY USE ONLY(Leave blank) & 2. REPORT DATE & 3. REPORT TYPE AND DATES COVERED
\end{tabular}

1. AGENCY USE ONLY(Leave blank) $\begin{gathered}\text { 2. REPORT DATE } \\ \text { October } 1997\end{gathered} \quad \begin{gathered}\text { 3. REPORT TYPE AND DA } \\ \text { Contractor Report }\end{gathered}$

4. TITLE AND SUBTITLE

Simulation of a Controlled Airfoil with Jets

5. FUNDING NUMBERS

C NAS1-19480

WU 505-90-52-01

6. AUTHOR(S)

Brian G. Allan

Maurice Holt

Andrew Packard

7. PERFORMING ORGANIZATION NAME(S) AND ADDRESS(ES)

Institute for Computer Applications in Science and Engineering

Mail Stop 403, NASA Langley Research Center

Hampton, VA 23681-0001

8. PERFORMING ORGANIZATION REPORT NUMBER

ICASE Report No. 97-55

9. SPONSORING/MONITORING AGENCY NAME(S) AND ADDRESS(ES)

National Aeronautics and Space Administration

Langley Research Center

Hampton, VA 23681-2199

10. SPONSORING/MONITORING AGENCY REPORT NUMBER

NASA CR-201750

ICASE Report No. 97-55

\section{SUPPLEMENTARY NOTES}

Langley Technical Monitor: Dennis M. Bushnell

Final Report

To appear in the Journal of Guidance, Control, and Dynamics

12a. DISTRIBUTION/AVAILABILITY STATEMENT

Unclassified-Unlimited

Subject Category 64

Distribution: Nonstandard

Availability: NASA-CASI (301)621-0390

13. ABSTRACT (Maximum 200 words)

Numerical simulations of a two-dimensional airfoil, controlled by an applied moment in pitch and an airfoil controlled by jets, were investigated. These simulations couple the Reynolds-averaged Navier-Stokes equations and Euler's equations of rigid body motion, with an active control system. Controllers for both systems were designed to track altitude commands and were evaluated by simulating a closed-loop altitude step response using the coupled system. The airfoil controlled by a pitching moment used an optimal state feedback controller. A closed-loop simulation, of the airfoil with an applied moment, showed that the trajectories compared very well with quasi-steady aerodynamic theory, providing a measure of validation. The airfoil with jets used a controller designed by robust control methods. A linear plant model for this system was identified using open-loop data generated by the nonlinear coupled system. A closed-loop simulation of the airfoil with jets, showed good tracking of an altitude command. This simulation also showed oscillations in the control input as a result of dynamics not accounted for in the control design. This research work demonstrates how computational fluid dynamics, coupled with rigid body dynamics, and a control law can be used to prototype control systems in problematic nonlinear flight regimes.

14. SUBJECT TERMS

Navier-Stokes equations, numerical simulation, feedback control, nonlinear flight regime, jets, coupled fluid dynamics, rigid body dynamics, controls

17. SECURITY CLASSIFICATION
OF REPORT
Unclassified

18. SECURITY CLASSIFICATION
OF THIS PAGE
Unclassified

19. SECURITY CLASSIFICATION Unclassified

\section{OF ABSTRACT}

\begin{tabular}{|c|}
\hline $\begin{array}{l}\text { 15. NUMBER OF PAGES } \\
20\end{array}$ \\
\hline $\begin{array}{r}\text { 16. PRICE CODE } \\
\text { A03 }\end{array}$ \\
\hline $\begin{array}{l}\text { 20. LIMITATION } \\
\text { OF ABSTRACT }\end{array}$ \\
\hline
\end{tabular}

